Cilt: 3

Sayı: 1

Sayfa: $49-57$

Kasım 2016

Volume: 3

Issue: 1

Page: $49-57$

November 2016

\section{Koordinat dönüşümünde EIV model klasik dengeleme yoluyla nasıl çözülür?}

\author{
Cüneyt Aydın* \\ Yıldız Teknik Üniversitesi, Harita Mühendisliği Bölümü, Jeodezi Anabilim Dalı, Esenler-Istanbul, Türkiye
}

\section{Özet}

Bir koordinat dönüşümü probleminde yalnız bir sistemin değil, her iki sistemin koordinatlar rasgele hatalı olabilir. Böylesi bir durumda ilgili problem, EIV model çerçevesinde ele alınır ve WTLS yöntemiyle çözülür. WTLS yönteminin uygulanmasında iki temel zorlukla karşılaşılır: (1) Çözüm için, EIV modelin katsayılar matrisinde geçen tüm elemanlara ilişkin de uygun bir kofaktör matris düşünülmelidir. Dönüşüm probleminin türüne bağlı olarak, katsayılar matrisindeki bazı elemanlar, iki ya da daha fazla kez aynı ya da farklı işaretli olarak geçtiği ve bazı elemanlar da hatasız olabildiği için, bu, her zaman kolay bir işlem değildir. Bu nedenle, modelin stokastik kısmını oluşturmak özel bir çabayı gerektirir. (2) WTLS çözümüne ilişkin eşitliklerin elde edilmesi, bilinen en küçük kareler dengelemesine göre oldukça karmaşıktır. Bu da, bazı nümerik ve istatistiki yöntem ve fikirlerin çözüme uyarlanmasını zorlaştırır. Söz konusu zorlukları ortadan kaldırmaya yönelik olarak, bu çalışmada, ilgili problemin çözümünün alışılageldik dengeleme yoluyla nasıl yapılacağı irdelenmektedir. Bu amaçla oluşturulan bir dengeleme işleminin WTLS çözümüne denk olduğu, matematiksel olarak gösterilmektedir. Söz konusu dengeleme işleminin çıkarımı, burada 2B Afin dönüşümü için çalışılmıştır, ancak, herhangi bir koordinat dönüşümüne kolayca uyarlanabilecek biçimde anlatılmıştır.

Anahtar Sözcükler

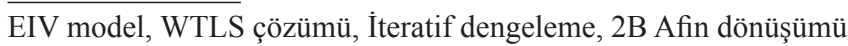

\section{Abstract \\ How to solve errors-in-variables model for coordinate transformations in a classical adjustment way?}

In a coordinate transformation problem, the coordinates of both systems may have random errors. In such a case, the corresponding problem is considered within the Errors-In-Variables (EIV) model and solved by the method of Weighted Total Least-Squares (WTLS). However there are two main difficulties while applying the WTLS method: (1) A proper cofactor matrix for all elements in the design matrix of the EIV model is also to be formed. This is sometimes confusing work because some elements may be repeated twice or more in the same or different signs and some elements may be error-free coefficients in the design matrix depending on the type of the transformation problem. Hence setting the stochastic part of the model needs a special effort. (2) The derivation of the equations of the WTLS solution is complicated in contrast to the least-squares adjustment. So it may not be easy work to adapt some numerical and statistical methods and ideas to the solution. In order to remove these difficulties this study discusses the solution of the corresponding problem in a classical adjustment way. It is shown mathematically that the adjustment procedure derived for this aim is equivalent to the WTLS solution. Although the procedure is examined here for 2D Affine transformation, it may be easily adapted to other coordinate transformation problems.

Keywords

EIV model, WTLS solution, Iterative adjustment, 2D Affine transformation

\footnotetext{
** Hakemli değerlendirme sürecinden geçerek, Jeodezi ve Jeoinformasyon Dergisi'nde yayına kabul edilen ve asıl yazı dili İngilizce olan "How to solve errors-in-variables model for coordinate transformations in a classical adjustment way?" başıılı eserin Türkçe diline çevirisidir. Çeviren; Cüneyt Aydın.
}

* Sorumlu Yazar: 


\section{Giriş}

Dengeleme problemlerinde, dengeleme modeline ilişkin katsayılar matrisi hatasız elemanlardan oluşur. Ancak, dengeleyici doğru, fonksiyon ve koordinat dönüşümü gibi problemlerde, katsayılar matrisinin bazı elemanları rasgele hatalarla yüklü olabilir. Böylesi bir durumda kestirim modeli, Errors-In-Variables (EIV) model olarak adlandirılan, "katsayıların da hatalı olduğu dengeleme modeli" altında oluşturulur (Golub ve Van Loan 1980; Carroll ve Ruppert 1996; van Huffel 2004). Gözlemlere ilişkin dolu bir kofaktör matrise sahip EIV modelin çözümü, Ağırlıklı Toplam En Küçük Kareler (WTLS) yöntemi ile gerçekleştirilir (Schaffrin ve Wieser 2008). Jeodezide söz konusu yöntem, genellikle, her iki sistem koordinatlarının rasgele hatalı olduğu koordinat dönüşümü problemlerinde uygulanır.

WTLS yöntemi, problemde geçen tüm hataların ağırlıklı karelerinin toplamının, bir koşul denklem sistemi altında minimum yapılması için oluşturulan bir Lagrange fonksiyonuna dayanır. Bu fonksiyonun çözümü için izlenen farklı yollar ile farklı iteratif algoritmalar oluşturulur. Fang (2011; 2013) ve Snow (2012), bu algoritmaları oldukça ayrıntılı olarak incelemektedirler. Aydın vd. (2014) ise, bu iteratif algoritmaları üç farklı grup altında sınıflandırmaktadır: (1) Genelleştirilmiş normal denklem temelli WTLS algoritması (Snow 2012); (2) en küçük kareler temelli WTLS algoritmas1 (Snow 2012; Amiri-Simkooei ve Jazaeri 2012) ve (3) geliştirilmiş WTLS algoritması (Tong et al. 2011). Problemin çözümü için başka çözüm yolları da vardır. Ancak bunlarla da benzer algoritmalar elde edilir. Örneğin, EIV model, bazı matris eşitlikleri kullanılarak, doğrusal olmayan Gauss-Helmert modele dönüştürülür (Schaffrin ve Snow 2010; Snow 2012). Bunun uygun bir biçimde en küçük kareler yöntemiyle çözümü, yukarıda sözü edilen ikinci algoritmanın eşitliklerini verir (bk. Bölüm 3).

Söz konusu algoritmalarla özdeş nümerik sonuçlar elde edilir. Ancak bunların uygulanmasında iki zorlukla karşılaş1lır: (1) Bu algoritmalarda, uygun bir modifikasyon işlemiyle gözlemlerin (koordinatların) kofaktör matrislerinden dönüştürülen özel bir kofaktör matrise ihtiyaç duyulur. Özellikle korelasyonlu gözlemler için bu matrisin elde edilmesi, özel bir çaba harcanmasını gerektirir; (2) WTLS algoritmalarının eşitlikleri, bilinen en küçük kareler dengeleme eşitliklerine göre oldukça karmaşıktır. Bu nedenle, bazı nümerik ve istatistiki yöntemlerin bu algoritmalara uyarlanması kolay bir işlem değildir. Söz konusu zorlukları ortadan kaldırmaya yönelik olarak, bu çalışmada, "TLS'nin genelleştirilmesi" (Neitzel 2010) ve "genel en küçük kareler yöntemi” (Ghilani ve Wolf 2006) birlikte ele alınarak, ilgili EIV probleminin klasik dengeleme yoluyla çözümü irdelenmektedir. Neitzel (2010), 2B benzerlik dönüşümü örneği üzerinden, Pope (1972)'nin uyarılarını göz önüne alarak, EIV probleminin alışılageldik (Gauss-Helmert türü) iteratif en küçük kareler dengelemesi biçiminde nasıl çözüleceğini göstermektedir. Söz konusu dengelemede, korelasyonsuz koordinatlara ilişkin kofaktör matrisi, herhangi bir modifikasyona tabi tutulmaksızın doğrudan kullanmaktadır. Yanı sıra bu çözüm, WTLS algoritmalarına göre daha açık eşitliklere sahiptir. Neitzel (2010), söz konusu iteratif dengeleme işleminin WTLS çözümüyle özdeş sonuç verdiğini nümerik olarak göstermektedir. Diğer yandan, Ghilani ve Wolf (2006), Neitzel (2010)'a benzer bir dengeleme yaklaşımıyla, genel en küçük kareler yönteminde EIV problemini çözmeye çalışmaktadır. Ancak bu yöntemde yanlış bir yol izlenmektedir: Dönüşüm problemine ilişkin fonksiyonel modelde doğrusallaştırma işlemi, "sistem koordinatlarına ve bilinmeyen parametrelere" göre yapılmaktadır. Bu yöntem ile WTLS çözümüne denk bir çözüm elde edebilmek için, doğrusallaştırmanın "koordinatların hatalarına ve bilinmeyen parametrelere" göre yapılması gerekmektedir. Söz konusu iki çalışma birlikte düşünülerek, EIV model çerçevesindeki her iki sistem koordinatlarının korelasyonlu olduğu koordinat dönüşümü probleminin çözümü için, bir iteratif dengeleme işlemi bu çalışmada ele alınmaktadır. Buna yönelik olarak, çalışmanın içeriği şöyledir: Bölüm 2, EIV model ve bunun en küçük kareler temelli algoritma ile WTLS çözümünü kısaca ele almaktadır. Bu WTLS algoritmasının doğrusal olmayan Gauss-Helmert modelinin çözümü biçimdeki alternatif çıkarım1, 2B Afin dönüşümü örneği üzerinden genel bir çerçevede Bölüm 3'de verilmektedir. Söz konusu problemin çözümüne ilişkin iteratif dengeleme işlemi, Bölüm 4'de irdelenmektedir. Bu bölümde ayrıca, ilgili iteratif dengeleme işleminin WTLS çözümüne denk olduğu, matematiksel olarak gösterilmektedir. Yanı sıra, iteratif kestirim işlemi sırasında bazı nümerik sorunlara neden olabilen büyük koordinatlar yerine, ötelenmiş koordinatlar ile nasıl çalışılacağı, aynı bölümde işlenmektedir. Sayısal bir örnek, Bölüm 5'de verilmektedir. Son bölümde ise çalışmanın sonuçları ele alınmaktadır.

\section{WTLS Çözümü}

\section{B Afin Dönüşümü için EIV Model}

Kartezyen $x y$ (hedef) ve $u v$ (başlangıç) koordinat sistemleri arasındaki standart 2B Afin dönüşümü aşağıdaki gibi tanımlanır;

$x=t_{1}+u k_{1}-v k_{2} ; y=t_{2}+u k_{3}+v k_{4}$

Burada, $t$ ve $k$, sirasiyla, (bilinmeyen) öteleme ve dönüşüm parametresini göstermektedir. $\mathrm{p}>3$ sayıdaki eşlenik noktanın $2 p(=n) \times 1$ boyutlu $\boldsymbol{y}=\left[\begin{array}{ll}\ldots x_{i} & y_{i} \ldots\end{array}\right]^{T}$ ve $\boldsymbol{s}=\left[\ldots u_{i} v_{i} \ldots\right]^{T}$ vektörlerinde tanımlı hedef ve başlangıç sistem koordinatları aşağıdaki gibi normal dağılmış rasgele hatalarla yüklü olsun:

$$
\begin{aligned}
\boldsymbol{e} & =\left[\begin{array}{l}
\boldsymbol{e}_{y} \\
\boldsymbol{e}_{s}
\end{array}\right] \\
& =\left[\ldots e_{x i} e_{y i} \ldots e_{u i} e_{v i} \ldots\right]^{T} \sim N\left(\left[\begin{array}{l}
\mathbf{0} \\
\mathbf{0}
\end{array}\right], \sigma^{2}\left[\begin{array}{cc}
\boldsymbol{Q}_{y} & \boldsymbol{Q}_{y s} \\
\boldsymbol{Q}_{s y} & \boldsymbol{Q}_{s}
\end{array}\right]=\sigma^{2} \boldsymbol{Q}_{\mathbf{e}}\right)
\end{aligned}
$$

Burada, $e$, rasgele hatayı; $\boldsymbol{e}_{y}$ ve $\boldsymbol{e}_{s}$ hedef ve başlangıç sistem koordinatlarının $n \times 1$ boyutlu rasgele hata vektörlerini; $\sigma^{2}$, (bilinmeyen) varyans bileşenini ve $\boldsymbol{Q}_{j}(j=y, s$ and $y s)$, rankı "rank $\boldsymbol{Q}_{j}=n(j=y, s$ için $)$ " olan kofaktör matrisi göstermektedir. $p$ sayıdaki eşlenik noktanın hatalı koordinatları için, model (1)'den, 


$$
\begin{aligned}
& x_{i}-e_{x i}=t_{1}+\left(u_{i}-e_{u i}\right) k_{1}-\left(v_{i}-e_{v i}\right) k_{2} \\
& y_{i}-e_{y i}=t_{2}+\left(u_{i}-e_{u i}\right) k_{3}+\left(v_{i}-e_{v i}\right) k_{4} \quad(i=1, \ldots, p)
\end{aligned}
$$

yazılır. $\mathrm{Bu}$, söz konusu dönüşüm problemi için geçerli EIV modelin fonksiyonel kısmıdır. Eşitlik (3) yeniden düzenlenerek, bu fonksiyonel kısmın daha yaygın bir biçimi,

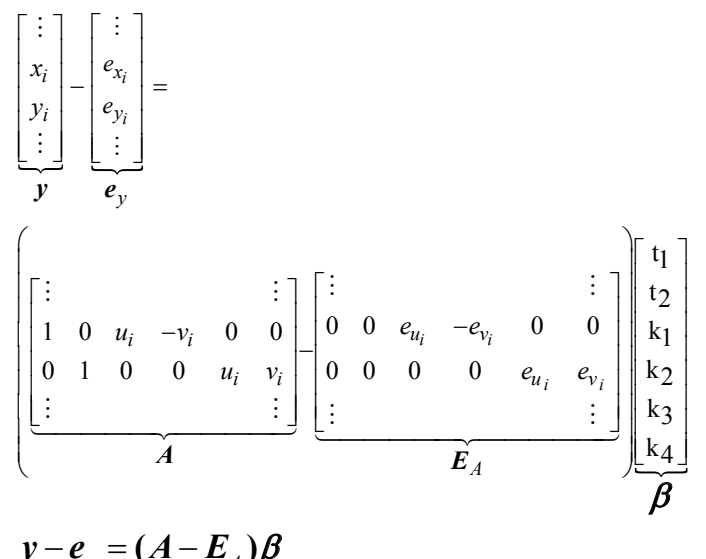

elde edilir. Burada, $\boldsymbol{A}, n \times 6$ boyutlu, data matrisi olarak adlandirılan katsayılar matrisi (bk. Snow 2012); $\boldsymbol{\beta}, 6 \times 1$ boyutlu bilinmeyen parametre vektörü ve $\boldsymbol{E}_{A}, n \times 6$ boyutlu hata matrisidir. EIV modelin stokastik kısmı için kovaryans matris $(\boldsymbol{C})$, Eşitlik (4)'de geçen $\boldsymbol{e}_{y}$ hata vektörü ve $\boldsymbol{E}_{A}$ hata matrisi için oluşturulur. Eşitlik (4)'den de görüldüğüu üzere, $\boldsymbol{E}_{A}$ hata matrisi, başlangıç sistem koordinatlarına ilişkin hataları içerir. Bir başka deyişle, bu hata matrisi, başlangıç sistem koordinatlarına ilişkin $\boldsymbol{e}$ hata vektörünün bir fonksiyonudur. Söz konusu ilişki, aşağıdaki biçimde gösterilebilir;

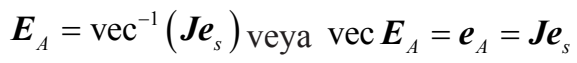

Burada, vec, soldan sağa doğru, bir matrisin her bir sütununun alta alta getirilmesini sağlayan bir lineer cebir operatörü; vec $^{-1}$, bir vektörden uygun bir matrisin elde edilmesini gerçekleştiren ters vec operatörü; $\boldsymbol{e}_{A}, \boldsymbol{A}$ matrisine ilişkin $6 n \times 1$ boyutlu hata vektörü $\left(\boldsymbol{e}_{A}=\mathrm{vec} \boldsymbol{E}_{A}\right)$ ve $\boldsymbol{J}, 6 n \times n$ boyutlu $\boldsymbol{e}_{s}^{\prime}$ den $\boldsymbol{e}_{A}$ 'ya dönüşümü sağlayan Jacobian matristir (bk. Ek A). Eşitlik (2) ve kofaktör yayılma kuralı düşünülerek $\gamma=\left[\boldsymbol{e}_{y} \boldsymbol{e}_{A}\right]^{T}$ hata vektörünün dağılımı, böylece,

$\boldsymbol{\gamma}=\left[\begin{array}{l}\boldsymbol{e}_{y} \\ \boldsymbol{e}_{A}\end{array}\right] \sim N\left(\left[\begin{array}{l}\mathbf{0} \\ \mathbf{0}\end{array}\right], \sigma^{2}\left[\begin{array}{cc}\boldsymbol{Q}_{y} & \boldsymbol{Q}_{y s} \boldsymbol{J}^{T} \\ \boldsymbol{J} \boldsymbol{Q}_{s y} & \boldsymbol{J} \boldsymbol{Q}_{s} \boldsymbol{J}^{T}\end{array}\right]=\sigma^{2}\left[\begin{array}{cc}\boldsymbol{Q}_{y} & \boldsymbol{Q}_{y A} \\ \boldsymbol{Q}_{A y} & \boldsymbol{Q}_{A}\end{array}\right]\right)(6)$

çıkar. Sonuçta, model (4) ve model (6) ile EIV model oluşturulur;

$$
\boldsymbol{y}-\boldsymbol{e}_{y}=\left(\boldsymbol{A}-\boldsymbol{E}_{A}\right) \boldsymbol{\beta}, \boldsymbol{C}_{\gamma}=\sigma^{2}\left[\begin{array}{ll}
\boldsymbol{Q}_{y} & \boldsymbol{Q}_{y A} \\
\boldsymbol{Q}_{A y} & \boldsymbol{Q}_{A}
\end{array}\right]=\sigma^{2} \boldsymbol{Q}_{\gamma}
$$

\section{WTLS Çözümü}

Model (7)'de geçen bilinmeyen parametrelerin kestirimi, WTLS yöntemi ile gerçekleştirilir (Schaffrin ve Wieser, 2008). Bu yöntem,
$\Omega=\left[\boldsymbol{e}_{y}^{T} \boldsymbol{e}_{A}^{T}\right]\left(\left[\begin{array}{ll}\boldsymbol{Q}_{y} & \boldsymbol{Q}_{y A} \\ \boldsymbol{Q}_{A y} & \boldsymbol{Q}_{A}\end{array}\right]\right)^{-1}\left[\begin{array}{l}\boldsymbol{e}_{y} \\ \boldsymbol{e}_{A}\end{array}\right]$

karesel biçiminin (bk. Fang 2011; Snow 2012), “ $\boldsymbol{y}-\boldsymbol{e}=\left(\boldsymbol{A}-\boldsymbol{E}_{A}\right) \boldsymbol{\beta}$ ” koşul denklemlerine göre minimum yapılmasına dayanır. Bu amaçla, “ $\boldsymbol{E}_{A} \boldsymbol{\beta}=\left(\boldsymbol{\beta}^{T} \otimes \boldsymbol{I}_{n}\right) \mathbf{e}_{A}$ ” vektör eşitliğ (Koch 1999) düşünülerek, aşağıdaki hedef (Lagrange) fonksiyonu oluşturulur;

$$
\begin{aligned}
\phi & =\Omega+2 \lambda^{T}\left(\boldsymbol{y}-\boldsymbol{e}_{y}-\boldsymbol{A} \boldsymbol{\beta}+\boldsymbol{E}_{A} \boldsymbol{\beta}\right) \\
& =\Omega+2 \boldsymbol{\lambda}^{T}\left(\boldsymbol{y}-\boldsymbol{e}_{y}-\boldsymbol{A} \boldsymbol{\beta}+\left(\boldsymbol{\beta}^{T} \otimes \boldsymbol{I}_{n}\right) \boldsymbol{e}_{A}\right)
\end{aligned}
$$

Burada, $\lambda, n \times 1$ boyutlu Lagrange çarpanları vektörü; $\otimes$ Kronecker çarpımı ve $\boldsymbol{I}_{n}, n \times n$ boyutlu birim matristir. $\phi$ fonksiyonunun $\boldsymbol{e}_{v}, \boldsymbol{e}_{A}, \boldsymbol{\beta}$ ve $\lambda$ 'ya göre kısmi türevlerinden elde edilen Euler koşul eşitliklerinden,

$$
\begin{aligned}
& \tilde{\boldsymbol{e}}_{A}=\left(\boldsymbol{Q}_{A y}-\boldsymbol{Q}_{A}\left(\hat{\boldsymbol{\beta}} \otimes \boldsymbol{I}_{n}\right)\right) \hat{\boldsymbol{\lambda}}, \tilde{\boldsymbol{E}}_{A}=\operatorname{vec}^{-1}\left(\tilde{\boldsymbol{e}}_{A}\right) \\
& \tilde{\boldsymbol{e}}_{y}=\left(-\boldsymbol{Q}_{y A}\left(\hat{\boldsymbol{\beta}} \otimes \boldsymbol{I}_{n}\right)+\boldsymbol{Q}_{y}\right) \hat{\lambda}
\end{aligned}
$$

$$
Q_{1} \hat{\lambda}=\boldsymbol{y}-\boldsymbol{A} \hat{\beta}
$$

$$
\begin{aligned}
\boldsymbol{Q}_{1} & =\boldsymbol{Q}_{y}-\boldsymbol{Q}_{y A}\left(\hat{\boldsymbol{\beta}} \otimes \boldsymbol{I}_{n}\right)-\left(\hat{\boldsymbol{\beta}} \otimes \boldsymbol{I}_{n}\right)^{T} \boldsymbol{Q}_{A y} \\
& +\left(\hat{\boldsymbol{\beta}} \otimes \boldsymbol{I}_{n}\right)^{T} \boldsymbol{Q}_{A}\left(\hat{\boldsymbol{\beta}} \otimes \boldsymbol{I}_{n}\right)
\end{aligned}
$$

$$
\left(\boldsymbol{A}-\tilde{\boldsymbol{E}}_{\mathrm{A}}\right)^{T} \hat{\boldsymbol{\lambda}}=\mathbf{0}
$$

çıkar (bk. Snow 2012). Burada, “〜” ve “^”, sırasıyla, predikte edilen ve kestirilen değerleri göstermektedir. (10c-d) eşitlikleri kullanılarak, aşağıdaki normal denklemlere ulaşılır;

$\left[\begin{array}{cc}\boldsymbol{Q}_{1} & \boldsymbol{A}-\tilde{\boldsymbol{E}}_{A} \\ \left(\boldsymbol{A}-\tilde{\boldsymbol{E}}_{\mathrm{A}}\right)^{T} & \boldsymbol{0}\end{array}\right]\left[\begin{array}{l}\hat{\lambda} \\ \hat{\boldsymbol{\beta}}\end{array}\right]=\left[\begin{array}{c}\boldsymbol{y}-\tilde{\boldsymbol{E}}_{A} \hat{\boldsymbol{\beta}} \\ 0\end{array}\right]$

$\hat{\lambda}$ çarpan vektörünün indirgenmesiyle,

$$
\left(\boldsymbol{A}-\tilde{\boldsymbol{E}}_{A}\right)^{T} \boldsymbol{Q}_{1}^{-1}\left(\boldsymbol{A}-\tilde{\boldsymbol{E}}_{A}\right) \hat{\boldsymbol{\beta}}=\left(\boldsymbol{A}-\tilde{\boldsymbol{E}}_{A}\right)^{T} \boldsymbol{Q}_{1}^{-1}\left(\boldsymbol{y}-\tilde{\boldsymbol{E}}_{A} \hat{\boldsymbol{\beta}}\right)
$$

bulunur. Son eşitliğin her iki yanı, $\hat{\boldsymbol{\beta}}$ parametre vektörünü içermektedir. $\mathrm{Bu}$ nedenle, doğrudan bir çözüm söz konusu değildir. Çözüm, iteratif olarak gerçekleştirilir. Bunun için, $\boldsymbol{\beta}$ ve $\boldsymbol{E}_{A}$ 'ya ilişkin başlangıç değerlerine ihtiyaç vardır: Yalnız hedef koordinatlarının hatalı olduğu varsayılarak, bu başlangıç değerleri şöyle belirlenir;

$$
\hat{\boldsymbol{\beta}}_{0}=\left(\boldsymbol{A}^{T} \boldsymbol{Q}_{y}^{-1} \boldsymbol{A}\right)^{-1} \boldsymbol{A}^{T} \boldsymbol{Q}_{y}^{-1} \boldsymbol{y} \quad, \tilde{\boldsymbol{E}}_{A, 0}=\mathbf{0}
$$

(10)-(13) eşitlikleri göz önüne alınarak, iteratif çözüm algoritması (en küçük kareler temelli algoritma), Ek B'de verildiği gibi tasarlanır (Snow 2012). $\hat{\boldsymbol{\beta}}$ parametre vektörü ve $\tilde{\boldsymbol{e}}_{A}$ hata vektörü, algoritmanın çözüme yakınsaması sonrasinda elde edilir. $\tilde{\boldsymbol{e}}_{y}$ hata vektörü ise, son iterasyondaki $\hat{\boldsymbol{\beta}}$ parametre vektörü kullanılarak, (10b) eşitliğinden doğrudan hesaplanır. Kestirilen parametrelerin kovaryans matrisi aşağ1daki biçimde elde edilir (Amiri-Simkooei ve Jazaeri 2012); 


$$
\begin{aligned}
& \boldsymbol{C}_{\hat{\beta}}=\hat{\sigma}^{2}\left[\left(\boldsymbol{A}-\tilde{\boldsymbol{E}}_{A}\right)^{T} \boldsymbol{Q}_{1}^{-1}\left(\boldsymbol{A}-\tilde{\boldsymbol{E}}_{A}\right)\right]^{-1}, \\
& \hat{\sigma}^{2}=\tilde{\Omega} /(n-6)
\end{aligned}
$$

Burada $\widetilde{\Omega}, \tilde{\boldsymbol{e}}_{y}$ ve $\tilde{\boldsymbol{e}}_{A}$ hata vektörlerine göre (8) eşitliğinden hesaplanan karesel biçimdir.

\section{WTLS Çözümü için Alternatif Çıkarım}

(7) EIV modeli aşağıdaki biçimde de yazılabilir;

$$
\begin{aligned}
& \left(\boldsymbol{A}-\boldsymbol{E}_{A}\right) \boldsymbol{\beta}-\left(\boldsymbol{y}-\boldsymbol{e}_{y}\right)=\boldsymbol{A} \beta+\underbrace{\left[\begin{array}{ll}
\boldsymbol{I}_{n} & -\left(\boldsymbol{\beta}^{T} \otimes \boldsymbol{I}_{n}\right)
\end{array}\right]}_{B} \underbrace{\left[\begin{array}{c}
\boldsymbol{e}_{y} \\
\boldsymbol{e}_{A}
\end{array}\right]}_{\gamma}-\boldsymbol{y} \\
& =\Psi(\boldsymbol{\beta}, \boldsymbol{\gamma})=\boldsymbol{A} \boldsymbol{\beta}+\boldsymbol{B} \boldsymbol{\gamma}-\boldsymbol{y}=\mathbf{0} \\
& \boldsymbol{C}_{\gamma}=\sigma_{0}^{2} \boldsymbol{Q}_{\gamma}
\end{aligned}
$$

Söz konusu yeni model, bir doğrusal olmayan GaussHelmert modelidir. Bu da ancak bir iteratif doğrusallaştırma işleminin düşünülmesi ile çözülebilir. $\boldsymbol{\beta}$ ve $\boldsymbol{\gamma}$, için ilk yaklaşık değerlere $\left(\boldsymbol{\beta}_{0}\right.$ ve $\left.\boldsymbol{\gamma}_{0}\right)$ ihtiyaç vardır (bk. (24) eşitliği). Bunların tespiti ile, model (15)'in ilk doğrusallaştırması, Taylor açınımı kullanılarak, aşağıdaki biçimde gerçekleştirilir;

$$
\psi(\boldsymbol{\beta}, \boldsymbol{\gamma})=\psi\left(\boldsymbol{\beta}_{0}, \boldsymbol{\gamma}_{0}\right)+\left.\frac{\partial \psi}{\partial \boldsymbol{\beta}^{T}}\right|_{0}\left(\boldsymbol{\beta}-\boldsymbol{\beta}_{0}\right)+\left.\frac{\partial \psi}{\partial \boldsymbol{\gamma}^{T}}\right|_{0}\left(\boldsymbol{\gamma}-\boldsymbol{\gamma}_{0}\right) \doteq \mathbf{0}
$$

Yukarıda geçen kısmi türevler ve $\psi\left(\boldsymbol{\beta}_{0}, \boldsymbol{\gamma}_{0}\right)$ yaklaşı fonksiyon değeri, Eşitlik (15)'den

$$
\begin{aligned}
& \left.\frac{\partial \psi}{\partial \boldsymbol{\beta}^{T}}\right|_{0}=\left(\boldsymbol{A}-\boldsymbol{E}_{A, 0}\right),\left.\frac{\partial \psi}{\partial \gamma^{T}}\right|_{0}=\boldsymbol{B}_{0}=\left[\begin{array}{ll}
\boldsymbol{I}_{n} & -\left(\boldsymbol{\beta}^{T} \otimes \boldsymbol{I}_{n}\right)
\end{array}\right] \\
& \psi\left(\boldsymbol{\beta}_{0}, \boldsymbol{\gamma}_{0}\right)=\left(\boldsymbol{A}-\boldsymbol{E}_{A, 0}\right) \boldsymbol{\beta}_{0}-\left(\boldsymbol{y}-\boldsymbol{e}_{y, 0}\right)
\end{aligned}
$$

biçiminde belirlenir. (17) eşitliği, (16)'da düşünülerek,

$$
\begin{aligned}
& \left(\boldsymbol{A}-\boldsymbol{E}_{A, 0}\right) \boldsymbol{\beta}_{0}-\left(\boldsymbol{y}-\boldsymbol{e}_{y, 0}\right)+\left(\boldsymbol{A}-\boldsymbol{E}_{A, 0}\right)\left(\boldsymbol{\beta}-\boldsymbol{\beta}_{0}\right)+\boldsymbol{B}_{0}\left(\boldsymbol{\gamma}-\boldsymbol{\gamma}_{0}\right) \doteq \mathbf{0}(18 \mathrm{a}) \\
& \left(\boldsymbol{A}-\boldsymbol{E}_{A, 0}\right) \boldsymbol{\beta}+\boldsymbol{B}_{0} \gamma=\left(\boldsymbol{y}-\boldsymbol{e}_{y, 0}\right)+\boldsymbol{B}_{0} \gamma_{0}
\end{aligned}
$$

çıkar. (18b) eşitliğinin sağ tarafı kapanma vektörünü gösterir. Eşitlik (17)'de geçen $\boldsymbol{B}_{0}$ matrisiyle bu kapanma vektörü için,

$$
\begin{aligned}
\left(\boldsymbol{y}-\boldsymbol{e}_{y, 0}\right)+\boldsymbol{B}_{0} \gamma_{0} & =\boldsymbol{y}-\boldsymbol{e}_{y, 0}+\boldsymbol{e}_{y, 0}-\left(\boldsymbol{\beta}_{0}{ }^{T} \otimes \boldsymbol{I}_{n}\right) \boldsymbol{e}_{A, 0} \\
& =\boldsymbol{y}-\boldsymbol{E}_{A, 0} \boldsymbol{\beta}_{0}
\end{aligned}
$$

bulunur. (18b) ve (19) eşitlikleriyle, sonuç olarak, aşağıdaki (doğrusal) Gauss-Helmert modeli elde edilir;

$$
\left(\boldsymbol{A}-\boldsymbol{E}_{A, 0}\right) \boldsymbol{\beta}+\boldsymbol{B}_{0} \boldsymbol{\gamma}=\boldsymbol{y}-\boldsymbol{E}_{A, 0} \boldsymbol{\beta}_{0}, \boldsymbol{C}_{\gamma}=\sigma_{0}^{2} \boldsymbol{Q}_{\gamma}
$$

Yukarıdaki model, doğrusal olmayan (15) modelinin ilk doğrusal halidir. En küçük kareler yöntemi ile çözülebilir. Koch (1999)'da verilen işlem sırası takip edilerek, $\boldsymbol{\beta}$ 'nın ilk kestirimi ve $\gamma^{\prime}$ nin ilk prediksiyonu şöyle bulunur;

$$
\hat{\boldsymbol{\beta}}_{1}=\left[\left(\boldsymbol{A}-\boldsymbol{E}_{A, 0}\right)^{T} \boldsymbol{Q}_{1,1}^{-1}\left(\boldsymbol{A}-\boldsymbol{E}_{A, 0}\right)\right]^{-1}\left(\boldsymbol{A}-\boldsymbol{E}_{A, 0}\right)^{T} \boldsymbol{Q}_{1,1}^{-1}\left(\boldsymbol{y}-\boldsymbol{E}_{A, 0} \boldsymbol{\beta}_{0}\right)
$$

$$
\begin{aligned}
\tilde{\gamma}_{1} & =\left[\begin{array}{l}
\tilde{\boldsymbol{e}}_{y, 1} \\
\tilde{\boldsymbol{e}}_{A, 1}
\end{array}\right] \\
& =\underbrace{\left(\left[\begin{array}{l}
\boldsymbol{Q}_{y}-\boldsymbol{Q}_{y A}\left(\boldsymbol{\beta}_{0} \otimes \boldsymbol{I}_{n}\right) \\
\boldsymbol{Q}_{A y}-\boldsymbol{Q}_{A}\left(\boldsymbol{\beta}_{0} \otimes \boldsymbol{I}_{n}\right)
\end{array}\right]\right)}_{\boldsymbol{Q}_{y} \boldsymbol{B}_{0}^{T}} \boldsymbol{Q}_{1,1}^{-1}\left(\boldsymbol{y}-\boldsymbol{E}_{A, 0} \boldsymbol{\beta}_{0}-\left(\boldsymbol{A}-\boldsymbol{E}_{A, 0}\right) \hat{\boldsymbol{\beta}}_{1}\right)
\end{aligned}
$$

Yukarıda geçen $\boldsymbol{Q}_{1,1}, \boldsymbol{B}_{0} \boldsymbol{\gamma}$ hata teriminin ağırlık katsay1ları matrisidir;

$$
\begin{aligned}
\boldsymbol{Q}_{1,1} & =\boldsymbol{B}_{0} \boldsymbol{Q}_{\gamma} \boldsymbol{B}_{0}^{T} \\
& =\boldsymbol{Q}_{y}-\boldsymbol{Q}_{y A}\left(\boldsymbol{\beta}_{0} \otimes \boldsymbol{I}_{n}\right)-\left(\boldsymbol{\beta}_{0} \otimes \boldsymbol{I}_{n}\right)^{T} \boldsymbol{Q}_{A y} \\
& +\left(\boldsymbol{\beta}_{0} \otimes \boldsymbol{I}_{n}\right)^{T} \boldsymbol{Q}_{A}\left(\boldsymbol{\beta}_{0} \otimes \boldsymbol{I}_{n}\right)
\end{aligned}
$$

(13) eşitliğine benzer biçimde, $\boldsymbol{\beta}$ ve $\boldsymbol{\gamma}$ için ilk yaklaşık değerler, aşağıdaki gibi varsayılabilir;

$$
\begin{aligned}
& \boldsymbol{\beta}_{0}=\hat{\boldsymbol{\beta}}_{0}=\left(\boldsymbol{A}^{T} \boldsymbol{Q}_{y}^{-1} \boldsymbol{A}\right)^{-1} \boldsymbol{A}^{T} \boldsymbol{Q}_{y}^{-1} \boldsymbol{y}, \\
& \boldsymbol{\gamma}_{0}=\hat{\gamma}_{0}=\left[\tilde{\boldsymbol{e}}_{y, 0} \tilde{\boldsymbol{e}}_{A, 0}\right]^{T}=\left[\begin{array}{ll}
\boldsymbol{0} & 0
\end{array}\right]^{T}, \boldsymbol{E}_{A, 0}=\tilde{\boldsymbol{E}}_{A, 0}=\mathbf{0}
\end{aligned}
$$

Böylece, (21) ve (23) eşitlikleri arasındaki çözümlerin, aslında, en küçük kareler temelli WTLS algoritmasının ilk iterasyon eşitlikleri olduğu görülür. Yani, doğrusal olmayan Gauss-Helmert modelinin çözümü, özdeş iteratif eşitlikler kullanılarak gerçekleştirilir. Buna göre, söz konusu çözümün WTLS çözümüne denk olduğu sonucu çıkar.

\section{EIV Model için İteratif Dengeleme İşlemi}

2B Afin dönüşüm örneği için Ghilani ve Wolf (2006)'da verilen 'genel en küçük kareler yöntemi, Eşitlik (3)'deki fonksiyonel modelin " $x y$ ve $u v$ koordinatlarına ve bilinmeyen parametrelere" göre doğrusallaştırılmasına ve buradan elde edilen Gauss-Helmert modelinin çözümüne dayanır. Ancak, söz konusu doğrusallaştırma işlemi, Neitzel (2010)'da gösterildiği üzere, "sistem koordinatlarının rasgele hataları ve bilinmeyen parametreler" için gerçekleştirilmelidir. Sözü edilen iki çalışma birlikte düşünülerek, korelasyonlu sistem koordinatları için EIV model çerçevesinde ele alınan dönüşüm probleminin bir iteratif dengeleme işlemi ile çözümü, burada açık olarak elde edilmektedir. Söz konusu dengeleme işleminin, ayrıca, en küçük kareler temelli WTLS algoritmasına denk olduğu da gösterilmektedir.

Şimdi, bilinmeyen $t_{1}$ ve $t_{2}$ öteleme parametrelerini Eşitlik (3)'den çıkaralım;

$$
\begin{aligned}
& F_{i}=x_{i}-e_{x i}-t_{1}=\left(u_{i}-e_{u i}\right) k_{1}-\left(v_{i}-e_{v i}\right) k_{2} \\
& G_{i}=y_{i}-e_{y i}-t_{2}=\left(u_{i}-e_{u i}\right) k_{3}+\left(v_{i}-e_{v i}\right) k_{4}(i=1, \ldots, p)
\end{aligned}
$$

Söz konusu $\boldsymbol{F}_{i}$ ve $\boldsymbol{G}_{i}$ fonksiyonları başlangıç sistemi koordinatlarının $e_{u}$ ve $e_{v}$ hatalarının ve de $k_{j}(j=1, \ldots, 4)$. bilinmeyen dönüşüm parametrelerinin doğrusal olmayan fonksiyonlarıdır. Bunlar, Taylor açınımı yardımıyla doğrusallaştırılabilir: Bunun için ilgili hatalar ve bilinmeyen parametreler iki kısma bölünür; 
$e_{u i}=e_{u i, 0}+\delta e_{u i}, e_{v i}=e_{v i, 0}+\delta e_{v i}, k_{j}=k_{j, 0}+\delta k_{j}$

$(j=1, \ldots, 4)$

Burada " $\delta$ " ifadesi, $(\ldots)_{0}$ yaklaşık değerinden olan küçük sapmayı göstermektedir. $F_{i}$ fonksiyonu, böylece,

$$
\begin{aligned}
F_{i} & =F_{i, 0}+\left.\frac{\partial F_{i}}{\partial e_{u i}}\right|_{0} \delta e_{u i}+\left.\frac{\partial F_{i}}{\partial e_{v i}}\right|_{0} \delta e_{v i}+\left.\frac{\partial F_{i}}{\partial k_{1}}\right|_{0} \delta k_{1}+\left.\frac{\partial F_{i}}{\partial k_{2}}\right|_{0} \delta k_{2} \\
& =\left(u_{i}-e_{u i, 0}\right) k_{1,0}-\left(v_{i}-e_{v i, 0}\right) k_{2,0}-k_{1,0} \delta e_{u i}+k_{2,0} \delta e_{v i} \\
& +\left(u_{i}-e_{u i, 0}\right) \delta k_{1}-\left(v_{i}-e_{v i, 0}\right) \delta k_{2}
\end{aligned}
$$

biçiminde yazılır. Son eşitlik, (26) eşitliğinin düşünülmesi ile, aşağıdaki biçime dönüşür;

$$
\begin{aligned}
F_{i} & =\left(u_{i}-e_{u i, 0}\right) k_{1}-\left(v_{i}-e_{v i, 0}\right) k_{2}-k_{1,0} \delta e_{u i}+k_{2,0} \delta e_{v i} \\
& =\left(u_{i}-e_{u i, 0}\right) k_{1}-\left(v_{i}-e_{v i, 0}\right) k_{2}-k_{1,0}\left(e_{u i}-e_{u i, 0}\right) \\
& +k_{2,0}\left(e_{v i}-e_{v i, 0}\right)
\end{aligned}
$$

(27b) eşitliğinin (25)'de göz önüne alınmasıyla,

$$
\begin{aligned}
x_{i}-\left(e_{u i, 0} k_{1,0}-e_{v i, 0} k_{2,0}\right) & =t_{1}+\left(u_{i}-e_{u i, 0}\right) k_{1}-\left(v_{i}-e_{v i, 0}\right) k_{2} \\
& +\left(e_{x i}-k_{1,0} e_{u i}+k_{2,0} e_{v i}\right)
\end{aligned}
$$

$(i=1, \ldots, p)$

bulunur. (25) eşitliğindeki $G_{i}$ fonksiyonundan da, yukarıdakine benzer biçimde, aşağıdaki eşitlik elde edilir;

$$
\begin{aligned}
y_{i}-\left(e_{u i, 0} k_{3,0}+e_{v i, 0} k_{4,0}\right) & =t_{2}+\left(u_{i}-e_{u i, 0}\right) k_{3}+\left(v_{i}-e_{v i, 0}\right) k_{4} \\
& +\left(e_{y i}-k_{3,0} e_{u i}-k_{4,0} e_{v i}\right)
\end{aligned}
$$

$(i=1, \ldots, p)$

(28) eşitliği, aşağıdaki biçimde de yazılabilecek düzeltme denklemlerini ifade eder;
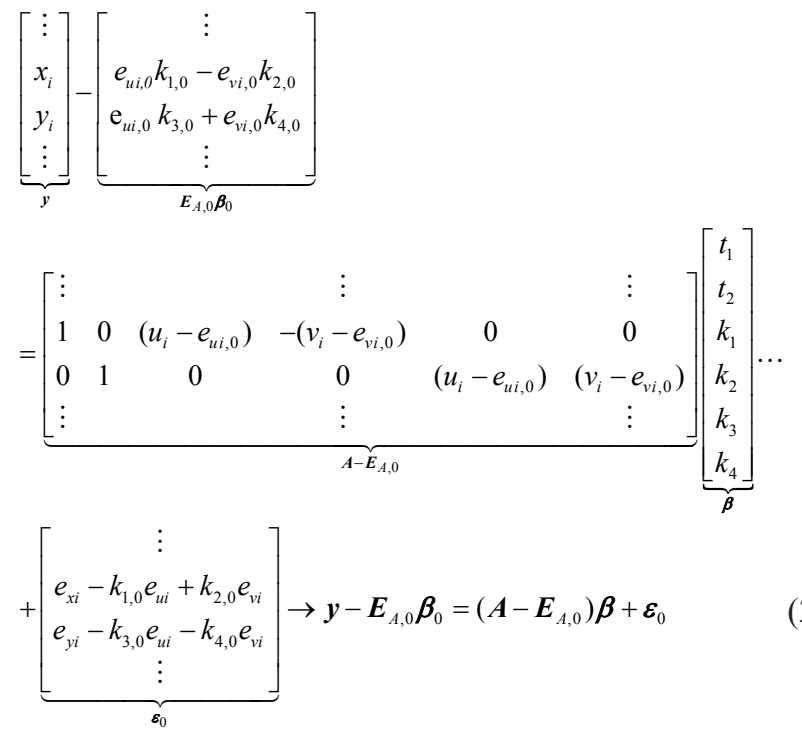

Burada, $\boldsymbol{\beta}_{0}$ ve $\boldsymbol{E}_{A, 0}(17)$ eşitliğinde olduğu gibi, yaklaşık parametre vektörünü ve hata matrisini gösterir. $\varepsilon_{0}$ ise aşağ1- daki biçimde ifade edilebilecek hata terimidir;

$\boldsymbol{\varepsilon}_{0}=\boldsymbol{D}_{0} \boldsymbol{e}=\left[\begin{array}{ll}\boldsymbol{I}_{n} & \boldsymbol{F}_{0}\end{array}\right]\left[\begin{array}{l}\boldsymbol{e}_{y} \\ \boldsymbol{e}_{s}\end{array}\right]=\left[\begin{array}{ll}\boldsymbol{I}_{n} & \left(\boldsymbol{I}_{n} \otimes \boldsymbol{K}_{0}\right)\end{array}\right]\left[\begin{array}{l}\boldsymbol{e}_{y} \\ \boldsymbol{e}_{s}\end{array}\right]$

$\boldsymbol{K}_{0}=\left[\begin{array}{cc}-k_{1,0} & k_{2,0} \\ -k_{3,0} & -k_{4,0}\end{array}\right]$

$\boldsymbol{\varepsilon}_{0}$ hata terimi, orijinal toplam hata vektörü olan e'ye bağlıdır. Eşitlik (2), (29) ve (30) ile aşağıdaki (doğrusal) GaussHelmert modeli elde edilir;

$$
\left(\boldsymbol{A}-\boldsymbol{E}_{A, 0}\right) \boldsymbol{\beta}+\boldsymbol{D}_{0} \boldsymbol{e}=\boldsymbol{y}-\boldsymbol{E}_{A, 0} \boldsymbol{\beta}_{0}, \boldsymbol{C}_{e}=\sigma_{0}^{2} \boldsymbol{Q}_{e}
$$

Bu, model (20) ile özdeştir. Çünkü, model (15)'de geçen B $\gamma$ hata terimi için,

$$
\begin{aligned}
\boldsymbol{B} \boldsymbol{\gamma} & =\left[\begin{array}{ll}
\boldsymbol{I}_{\mathrm{n}} & -\left(\boldsymbol{\beta}^{T} \otimes \boldsymbol{I}_{n}\right)
\end{array}\right]\left[\begin{array}{l}
\boldsymbol{e}_{y} \\
\boldsymbol{J} \boldsymbol{e}_{s}
\end{array}\right] \\
& =\left[\begin{array}{ll}
\boldsymbol{I}_{n} & -\left(\boldsymbol{\beta}^{T} \otimes \boldsymbol{I}_{n}\right) \boldsymbol{J}
\end{array}\right] \underbrace{\left[\begin{array}{l}
\boldsymbol{e}_{y} \\
\boldsymbol{e}_{s}
\end{array}\right]}_{\boldsymbol{e}} \\
& =\underbrace{\left[\begin{array}{ll}
\boldsymbol{I}_{n} & \left(\boldsymbol{I}_{n} \otimes \boldsymbol{K}\right)
\end{array}\right]}_{\boldsymbol{D}} \underbrace{\left[\begin{array}{c}
\boldsymbol{e}_{y} \\
\boldsymbol{e}_{s}
\end{array}\right]}_{\boldsymbol{e}}=\boldsymbol{D} \boldsymbol{e}
\end{aligned}
$$

eşitliği geçerlidir. Buna göre, (15) eşitliği ile verilen doğrusal olmayan Gauss-Helmert modelinin orijinal $\boldsymbol{e}$ hata vektörüne göre yazılabileceği ortaya çıkar;

$\Psi(\boldsymbol{\beta}, \boldsymbol{e})=\left(\boldsymbol{A}-\boldsymbol{E}_{A}\right) \boldsymbol{\beta}-\left(\boldsymbol{y}-\boldsymbol{e}_{y}\right)=\boldsymbol{A} \boldsymbol{\beta}+\boldsymbol{D} \boldsymbol{e}-\boldsymbol{y}=\mathbf{0}$

$\boldsymbol{C}_{e}=\sigma_{0}^{2} \boldsymbol{Q}_{e}$

$\mathrm{Bu}$ modelin ilk doğrusallaştırma sonucu, (28) ve (31) arasındaki eşitliklerde verilmişti. Bu da, aynı biçimde iteratif olarak çözülür, ancak burada, $\boldsymbol{e}$ orijinal hata vektörü predikte edilir. Örneğin, ilk iterasyon için (31) modelinin çözümü,

$$
\begin{aligned}
\tilde{\boldsymbol{e}}_{1} & =\left[\begin{array}{c}
\tilde{\boldsymbol{e}}_{y, 1} \\
\tilde{\boldsymbol{e}}_{s, 1}
\end{array}\right] \\
& =\left(\left[\begin{array}{c}
\boldsymbol{Q}_{y}+\boldsymbol{Q}_{y s} \boldsymbol{F}_{0}^{T} \\
\boldsymbol{Q}_{s y}+\boldsymbol{Q}_{s} \boldsymbol{F}_{0}^{T}
\end{array}\right]\right) \boldsymbol{Q}_{1,1}^{-1}\left(\boldsymbol{y}-\boldsymbol{E}_{A, 0} \boldsymbol{\beta}_{0}-\left(\boldsymbol{A}-\boldsymbol{E}_{A, 0}\right) \hat{\boldsymbol{\beta}}_{1}\right)
\end{aligned}
$$

prediksiyonunu verir. Burada geçen $\hat{\boldsymbol{\beta}}_{1}, \quad$ (21) eşitliğindeki kestirim değeri ve $\boldsymbol{Q}_{1,1}$ matrisi de “ $\boldsymbol{\varepsilon}_{0}=\boldsymbol{D}_{0} \boldsymbol{e}$ " hata terimine ilişkin kofaktör matristir. Bu kofaktör matris, (23) eşitliğindekine denktir. Ancak burada, özdeş olarak aşağıdaki biçimde elde edilir;

$\boldsymbol{Q}_{1,1}=\boldsymbol{D}_{0} \boldsymbol{Q}_{e} \boldsymbol{D}_{0}^{T}=\boldsymbol{Q}_{y}+\boldsymbol{Q}_{y s} \boldsymbol{F}_{0}^{T}+\boldsymbol{F}_{0} \boldsymbol{Q}_{s y}+\boldsymbol{F}_{0} \boldsymbol{Q}_{s} \boldsymbol{F}_{0}^{T}$

$\boldsymbol{E}_{A}$ hata matrisinin prediksiyonu, Eşitlik (3)'den 


$$
\tilde{\boldsymbol{E}}_{A, 1}=\left[\begin{array}{cccccc}
\vdots & & & & & \vdots \\
0 & 0 & \tilde{e}_{u_{i}, 1} & -\tilde{e}_{v_{i}, 1} & 0 & 0 \\
0 & 0 & 0 & 0 & \tilde{e}_{u_{i}, 1} & \tilde{e}_{v_{i}, 1} \\
\vdots & & & & & \vdots
\end{array}\right]
$$

şeklinde, (34) eşitliğine göre predikte edilen $\tilde{\boldsymbol{e}}_{s, 1}=\left[\ldots \tilde{e}_{u i, 1} \tilde{e}_{v i, 1} \ldots\right]^{T}$ hata vektöründeki elemanlara göre oluşturulur.

(24) ve (31) arasındaki eşitlikler, söz konusu problemin çözümünün klasik dengeleme işlemine benzer biçimde nasıl gerçekleştirileceğini göstermektedir. Doğal olarak bu, bir iteratif işlemdir. WTLS algoritmalarına benzer biçimde, bu iteratif dengeleme işlemi de, Şekil 1'de olduğu gibi, bir iteratif algoritma olarak verilebilir. Eşitlik (32) ve (33), halihazırda bu dengeleme işlemi ile önceki bölümde verilen çözümün denk olduğunu gösterir. Yani, en küçük kareler temelli WTLS algoritmasından farklı bir algoritma değildir. Ancak burada, $\boldsymbol{A}$ ve $\boldsymbol{y}$ 'ye ilişkin kofaktör matrisler $\left(\boldsymbol{Q}_{y}, \boldsymbol{Q}_{A}\right.$ ve $\left.\boldsymbol{Q}_{y A}=\boldsymbol{Q}_{A y}{ }^{T}\right)$ yerine, doğrudan koordinatların kofaktör matrisleri $\left(\boldsymbol{Q}_{y}, \boldsymbol{Q}_{s}\right.$ ve $\left.\boldsymbol{Q}_{y s}=\boldsymbol{Q}_{s y}{ }^{T}\right)$ kullanılmaktadır.

$\hat{\boldsymbol{\beta}}_{0}$ ve $\tilde{\boldsymbol{E}}_{A, 0}$ başlangıļ değerlerini tespit et (bk. (13) eşitliğgi).

$j=1,2 \ldots$ için tekrarla:

$$
\begin{aligned}
& \boldsymbol{K}_{j-1}=\left[\begin{array}{cc}
-\hat{k}_{1, j-1} & \hat{k}_{2, j-1} \\
-\hat{k}_{3, j-1} & -\hat{k}_{4, j-1}
\end{array}\right], \boldsymbol{F}_{j-1}=\left(\boldsymbol{I}_{n} \otimes \boldsymbol{K}_{j-1}\right), \\
& \boldsymbol{Q}_{1, j}=\boldsymbol{Q}_{y}+\boldsymbol{Q}_{y s} \boldsymbol{F}_{j-1}{ }^{T}+\boldsymbol{F}_{j-1} \boldsymbol{Q}_{s y}+\boldsymbol{F}_{j-1} \boldsymbol{Q}_{s} \boldsymbol{F}_{j-1}^{T}, \boldsymbol{P}_{1, j}=\boldsymbol{Q}_{1, j}^{-1} \\
& \tilde{\boldsymbol{A}}_{j}=\boldsymbol{A}-\tilde{\boldsymbol{E}}_{A, j-1}, \tilde{\boldsymbol{y}}_{j}=\boldsymbol{y}-\tilde{\boldsymbol{E}}_{A, j-1} \hat{\boldsymbol{\beta}}_{j-1}, \\
& \hat{\boldsymbol{\beta}}_{j}=\left(\tilde{\boldsymbol{A}}_{j}^{T} \boldsymbol{P}_{1, j} \tilde{\boldsymbol{A}}_{j}\right)^{-1} \tilde{\boldsymbol{A}}_{j}^{T} \boldsymbol{P}_{1, j} \tilde{\boldsymbol{y}}_{j} \\
& \tilde{\boldsymbol{e}}_{s, j}=\left(\boldsymbol{Q}_{s y}+\boldsymbol{Q}_{s} \boldsymbol{F}_{j-1}{ }^{T}\right) \boldsymbol{P}_{1, j}\left(\tilde{\boldsymbol{y}}-\tilde{\boldsymbol{A}}_{j} \hat{\boldsymbol{\beta}}_{j}\right), \tilde{\boldsymbol{E}}_{A, j} \text { matrisini, (36)'daki }
\end{aligned}
$$

gibi oluştur.

“ $\max \left(\left|\hat{\boldsymbol{\beta}}_{j}-\hat{\boldsymbol{\beta}}_{j-1}\right|\right)$ ” karşılaştırma değerinden küçük oluncaya dek iterasyona devam et.

Şekil 1. 2B Afin dönüşümünde korelasyonlu koordinatlar için iteratif dengeleme işlemi

İteratif dengeleme sonucunda parametre vektörü $\hat{\boldsymbol{\beta}}$ elde edilir. $\tilde{\boldsymbol{e}}$ orijinal toplam hata vektörü ise, iterasyonun çözüme yakınsaması sonrasında, Eşitlik (34) kullanılarak hesaplanır. Eşitlik (8)'de verilen $\tilde{\Omega}$ karesel biçimi, $\tilde{\boldsymbol{e}}$ hata vektörü kullanılarak, “ $\tilde{\Omega}=\tilde{\boldsymbol{e}}^{\mathrm{T}} \boldsymbol{Q}_{\mathrm{e}}^{-1} \tilde{\boldsymbol{e}}$ ”. şeklinde elde edilir. Dönüşümde

Çizelge 1. Hedef ve başlangıç sisteminde nokta koordinatları ve bunların ağırlıkları (Aydın vd. 2014)

\begin{tabular}{lcccc} 
& \multicolumn{5}{c}{ Hedef Sistem } \\
\cline { 2 - 5 } & $\mathrm{x}(\mathrm{m})$ & $\mathrm{y}(\mathrm{m})$ & $\mathrm{P}_{\mathrm{x}}\left(\mathrm{m}^{-2}\right)$ & $\mathrm{P}_{\mathrm{y}}\left(\mathrm{m}^{-2}\right)$ \\
\hline 1 & 4527754,612 & 434244,302 & 1,0 & 2,0 \\
\hline 2 & 4529097,150 & 432427,995 & 5,0 & 2,0 \\
\hline 3 & 4537389,003 & 434023,394 & 10,0 & 1,0 \\
\hline 4 & 4533316,751 & 429750,773 & 5,0 & 2,0 \\
\hline 5 & 4534306,216 & 426390,182 & 4,0 & 0,5 \\
\hline 6 & 4530615,243 & 427898,173 & 4,0 & 10,5 \\
\hline
\end{tabular}

geçen parametrelerin kovaryans matrisi (14) eşitliğinden hesaplanır.

\section{Nümerik Sorunların Giderilmesi}

Dönüşümde kullanılan jeodezik koordinatlar genellikle büyük sayı değerleridir. Bu, en küçük kareler dengelemesinden bilindiği üzere, normal denklemlerin çözümü sırasında bazı nümerik hatalara neden olur. Bunu gidermek için, $\boldsymbol{A}$ matrisinin elemanları ve gözlemler uygun şekilde ötelenir ve ölçeklendirilir. Burada, böylesi bir sorunun EIV modelden nasıl giderileceği açıklanmaktadır.

$t_{1}$ ve $t_{2}$ öteleme parametreleri için aşağıdaki eşitlikler düşünülebilir;

$t_{1}=x_{s}-u_{s} k_{1}+v_{s} k_{2}+\delta t_{1}$ ve $t_{2}=y_{s}-u_{s} k_{3}-v_{s} k_{4}+\delta t_{2}$

Burada, $\left(x_{s}, y_{s}\right)$ ve $\left(u_{s}, v_{s}\right)$ hedef ve başlangıç sisteminin ağırlık merkezi koordinatları ve $\delta t_{1}$ ve $\delta t_{2}$ ilgili öteleme parametrelerine ilişkin küçültülmüş bilinmeyenlerdir. Eşitlik (37), (28) eşitliğinde göz önüne alınırsa,

$$
\begin{aligned}
\Delta x_{i}-\left(e_{u i, 0} k_{1,0}-e_{v i, 0} k_{2,0}\right) & =\delta t_{1}+\left(\Delta u_{i}-e_{u i, 0}\right) k_{1}-\left(\Delta v_{i}-e_{v i, 0}\right) k_{2} \\
& +\left(e_{x i}-k_{1,0} e_{u i}+k_{2,0} e_{v i}\right)
\end{aligned}
$$

$(i=1, \ldots, p)$

$$
\begin{aligned}
\Delta y_{i}-\left(e_{u i, 0} k_{3,0}+e_{v i, 0} k_{4,0}\right)= & \delta t_{2}+\left(\Delta u_{i}-e_{u i, 0}\right) k_{3}+\left(\Delta v_{i}-e_{v i, 0}\right) k_{4} \\
& +\left(e_{y i}-k_{3,0} e_{u i}-k_{4,0} e_{v i}\right)
\end{aligned}
$$

$(i=1, \ldots, p)$

çıkar. $\Delta^{\prime} l_{1}$ ifadeler, ağırlık merkezine ötelenmiş koordinatlardir;

$\Delta x_{i}=x_{i}-x_{s}, \Delta y_{i}=y_{i}-y_{s}, \Delta u_{i}=u_{i}-u_{s}, \Delta v_{i}=v_{i}-v_{s}$

Eşitlik (38), çözümde orijinal koordinatlar yerine, ötelenmiş koordinatların kullanılabileceğini gösterir. Böylesi bir çözümde, önceki bölümde verilen algoritmaya ilişkin data matrisi $(\boldsymbol{A})$, hedef sistem koordinat vektörü $(\boldsymbol{y})$, ve bilinmeyen parametre vektörü $(\boldsymbol{\beta})$, aşağıdaki gibi ele alınır;

$\boldsymbol{A}=\left[\begin{array}{cccccc}\vdots & & & & & \vdots \\ 1 & 0 & \Delta u_{i} & -\Delta v_{i} & 0 & 0 \\ 0 & 1 & 0 & 0 & \Delta u_{i} & \Delta v_{i} \\ \vdots & & & & & \vdots\end{array}\right]$,

$\boldsymbol{y}=\left[\begin{array}{c}\vdots \\ \Delta x_{i} \\ \Delta y_{i} \\ \vdots\end{array}\right], \quad \boldsymbol{\beta}=\left[\begin{array}{c}\delta t_{1} \\ \delta t_{2} \\ k_{1} \\ k_{2} \\ k_{3} \\ k_{4}\end{array}\right]$

\begin{tabular}{cccc}
\multicolumn{4}{c}{ Başlangı̧ Sistemi } \\
\hline $\mathrm{u}(\mathrm{m})$ & $\mathrm{v}(\mathrm{m})$ & $\mathrm{P}_{\mathrm{u}}\left(\mathrm{m}^{-2}\right)$ & $\mathrm{P}_{\mathrm{v}}\left(\mathrm{m}^{-2}\right)$ \\
\hline$-12681,216$ & $-11115,112$ & 30,0 & 10,0 \\
\hline$-10849,480$ & $-9793,890$ & 4,0 & 20,0 \\
\hline$-12348,250$ & $-1484,610$ & 50,0 & 1,6 \\
\hline$-8123,500$ & $-5605,860$ & 50,0 & 2,4 \\
\hline$-4751,710$ & $-4655,920$ & 1,3 & 3,2 \\
\hline$-6302,628$ & $-8328,789$ & 1,4 & 36,0 \\
\hline
\end{tabular}


Çizelge 2. Ötelenmiş koordinatlar

\begin{tabular}{lcccc}
\hline $\mathrm{NN}$ & $\Delta \mathrm{x}(\mathrm{m})$ & $\Delta \mathrm{y}(\mathrm{m})$ & $\Delta \mathrm{u}(\mathrm{m})$ & $\Delta \mathrm{v}(\mathrm{m})$ \\
\hline 1 & $-4325,2172$ & 3455,1655 & $-3505,0853$ & $-4284,4152$ \\
\hline 2 & $-2982,6792$ & 1638,8585 & $-1673,3493$ & $-2963,1932$ \\
\hline 3 & 5309,1738 & 3234,2575 & $-3172,1193$ & 5346,0868 \\
\hline 4 & 1236,9218 & $-1038,3635$ & 1052,6307 & 1224,8368 \\
\hline 5 & 2226,3868 & $-4398,9545$ & 4424,4207 & 2174,7768 \\
\hline 6 & $-1464,5862$ & $-2890,9635$ & 2873,5027 & $-1498,0922$ \\
\hline
\end{tabular}

\section{Sayısal Uygulama}

İteratif dengeleme işlemini 2B Afin dönüşümü probleminde uygulayabilmek için, burada Aydın vd. (2014)'de verilen veri seti kullanılmaktadır. Bu veri seti, 6 noktanın iki sistemdeki koordinatlarını ve ağırlıklarını içermektedir (Çizelge 1).

\section{Hesaplama adımlarl:}

i) Eşitlik (39)'da gösterildiği üzere, söz konusu koordinatlar, ağırlık merkezine ötelenmiştir (Çizelge 2).

ii) $\boldsymbol{A}$ katsayılar matrisi ve $\boldsymbol{y}$ vektörü, (40) eşitliğinde gösterildiği gibi, Çizelge 2 'deki ötelenmiş koordinatlara göre düzenlenmiştir:

$$
\begin{aligned}
\boldsymbol{A} & =\left[\begin{array}{cccccc}
1 & 0 & -3505.0853 & 4284.4152 & 0 & 0 \\
0 & 1 & 0 & 0 & -3505.0853 & -4284.4152 \\
\vdots & \vdots & \vdots & \vdots & \vdots & \vdots
\end{array}\right] \\
\boldsymbol{y} & =\left[\begin{array}{c}
-4325.2172 \\
3455.1655 \\
\vdots
\end{array}\right](\mathrm{m})
\end{aligned}
$$

iii) Koordinatların kofaktör matrisleri, Çizelge 1'de verilen ağırlıklar kullanılarak şöyle hesaplanmıştır:

$\boldsymbol{Q}_{y}=\boldsymbol{P}_{y}^{-1}, \boldsymbol{Q}_{s}=\boldsymbol{P}_{s}^{-1} ;$

$\boldsymbol{P}_{y}=\operatorname{diag}\left(P_{x 1} P_{y 1} \ldots P_{x 6} P_{y 6}\right), \boldsymbol{P}_{s}=\operatorname{diag}\left(P_{u 1} P_{v 1} \ldots P_{u 6} P_{v 6}\right)$

İki sistem koordinatları korelasyonsuz olduğu için, $\boldsymbol{Q}_{y s}=\boldsymbol{Q}_{s y}=\mathbf{0}$ alınmıştır.

iv) İteratif dengeleme işlemine başlamak için, parametre vektörünün kestirimi “ $\hat{\boldsymbol{\beta}}_{0}=\left(\boldsymbol{A}^{T} \boldsymbol{Q}_{y}^{-1} \boldsymbol{A}\right)^{-1} \boldsymbol{A}^{T} \boldsymbol{Q}_{y}^{-1} \boldsymbol{y}$ ” şeklinde gerçekleştirilmiş ve hata matrisi için “ $\tilde{\boldsymbol{E}}_{A, 0}=\mathbf{0}$ ”. öngörül-

Çizelge 3. En küçük kareler (EKK) kestirim değerleri: iteratif dengeleme işleminin başlangıç değerleri

\begin{tabular}{cc}
\hline Parametre & EKK Kestrimi \\
$k_{1}$ & 0,011647225402 \\
$k_{2}$ & $-1,000003341129$ \\
$k_{3}$ & $-0,999994105682$ \\
$k_{4}$ & 0,011640379341 \\
$\delta t_{1}(m)$ & $-0,006306254073$ \\
$\delta t_{2}(m)$ & $-0,024925406785$ \\
\hline$t_{1}-4539017(m)$ & 0,418978172400 \\
$t_{2}-421692(m)$ & 0,546897262570 \\
\hline
\end{tabular}

müştür. Bu ilk adımda kestirilen parametreler, Çizelge 3'de verilmektedir. Ağırlık merkezine ötelenmiş koordinatlar kullanıldığı için, $\hat{\boldsymbol{\beta}}_{0}$ vektörü, Eşitlik (38)'e göre, " $\delta t_{1}$ ve $\delta t_{2}$ " bilinmeyenlerini içermektedir. (Not: $t_{1}$ ve $t_{2}$ öteleme parametreleri (37) eşitliğinden elde edilir.)

Yukarıda verilen başlangıç değerleri ile $\boldsymbol{F}_{0}$ Jacobian matrisi, aşağıdaki gibi oluşturulmuştur:

$$
\begin{aligned}
& \boldsymbol{F}_{0}=\left[\begin{array}{ccccc}
-\hat{k}_{1,0} & \hat{k}_{2,0} & \cdots & 0 & 0 \\
-\hat{k}_{3,0} & -\hat{k}_{4,0} & \cdots & 0 & 0 \\
\vdots & \vdots & \ddots & \vdots & \vdots \\
0 & 0 & \cdots & -\hat{k}_{1,0} & \hat{k}_{2,0} \\
0 & 0 & \cdots & -\hat{k}_{3,0} & -\hat{k}_{4,0}
\end{array}\right] \\
& =\left[\begin{array}{ccccc}
-0.0116 \ldots & -1.0000 \ldots & \cdots & 0 & 0 \\
0.9999 \ldots & -0.0116 \ldots & \cdots & 0 & 0 \\
\vdots & \vdots & \ddots & \vdots & \vdots \\
0 & 0 & \cdots & -0.0116 \ldots & -1.0000 \ldots \\
0 & 0 & \cdots & 0.9999 \ldots & -0.0116 \ldots
\end{array}\right]
\end{aligned}
$$

$\mathrm{Bu}$ matris, her iterasyon sonrasında elde edilen öteleme parametrelerinin yeni değerlerine göre diğer iterasyon için yeniden oluşturulur.

v) Şekil 1'de verilen iteratif dengeleme işlemine göre çözüm gerçekleştirilmiştir. Yakınsama denetimi için karşılaştırma değeri $\varepsilon=10^{-12}$ olarak seçilmiştir. Çözüme üçüncü iterasyonda ulaşılmıştır (Çizelge 4). Bu noktada şunu ifade etmekte yarar vardır: $\delta t_{1}$ ve $\delta t_{2}$ kestirim değerleri, ikinci ve üçüncü iterasyonda farklı olmalarına karşın, esas belirlenmek istenen $t_{1}$ ve $t_{2}$ öteleme parametrelerinin (37) eşitliğinden hesaplanan kestirim değerlerinin, söz konusu iterasyonlarda özdeş olduğu görülmektedir. Buradan, yakınsama denetiminin, arÇizelge 4. Iteratif dengeleme işleminde her bir iterasyonda elde edilen parametre kestirim değerleri

\begin{tabular}{cccc}
\hline Parametre & ilk iterasyon & İkinci Iterasyon & Üçüncü Iterasyon \\
\hline $\mathrm{k}_{1}$ & 0,011651721701 & 0,011651721608 & 0,011651721608 \\
\hline $\mathrm{k}_{2}$ & $-0,999998393160$ & $-0,999998393604$ & $-0,999998393604$ \\
\hline $\mathrm{k}_{3}$ & $-0,999985854949$ & $-0,999985855098$ & $-0,999985855098$ \\
\hline $\mathrm{k}_{4}$ & 0,011637345550 & 0,011637345558 & 0,011637345558 \\
\hline$\delta \mathrm{t}_{1}(\mathrm{~m})$ & 0,002427902022 & 0,002428130895 & 0,002428130884 \\
\hline$\delta \mathrm{t}_{2}(\mathrm{~m})$ & $-0,010194003949$ & $-0,010194180848$ & $-0,010194180847$ \\
\hline $\mathrm{t}_{1}-4539017(\mathrm{~m})$ & 0,435172882900 & 0,435175295000 & 0,435175295000 \\
\hline $\mathrm{t}_{2}-421692(\mathrm{~m})$ & 0,616615561420 & 0,616614076890 & 0,616614076890 \\
\hline
\end{tabular}


Çizelge 5. Iki sistem koordinatlarının hata prediksiyon değerleri

\begin{tabular}{|c|c|c|c|c|}
\hline \multirow{2}{*}{ NN } & \multicolumn{2}{|c|}{ Hedef Sistem } & \multicolumn{2}{|c|}{ Başlangıç Sistemi } \\
\hline & $e_{x}(m)$ & $e_{y}(m)$ & $e_{u}(m)$ & $e_{v}(m)$ \\
\hline 1 & 0,026335508457 & $-0,000806861724$ & $-0,000064018488$ & $-0,002631668669$ \\
\hline 2 & 0,003436019974 & 0,017848736298 & 0,008874197479 & $-0,000879774805$ \\
\hline 3 & 0,007442742337 & $-0,021129326553$ & $-0,000439924706$ & $-0,046363384087$ \\
\hline 4 & $-0,058543186238$ & 0,009588529504 & 0,000451748646 & 0,121871787879 \\
\hline 5 & 0,026284431422 & 0,076344315865 & 0,028420448062 & $-0,032994306058$ \\
\hline 6 & 0,017408793497 & $-0,006695584718$ & $-0,050795724831$ & $-0,001911580955$ \\
\hline
\end{tabular}

dişık iterasyonlarda " $k_{1}, \ldots, k_{4}$ " ve " $t_{1}$ ve $t_{2}$ " parametre kestirimlerine göre yapılması gerektiği sonucu çıkmaktadır.

Başlangıç ve hedef sistem koordinatlarına ilişkin $\tilde{\boldsymbol{e}}_{s}$ ve $\tilde{\boldsymbol{e}}_{y}$ hata vektörleri aşağıdaki şekilde hesaplanmıştır (bk. Eşitlik (34)):

$$
\begin{aligned}
& \tilde{\boldsymbol{e}}_{s}=\boldsymbol{Q}_{s} \boldsymbol{F}_{2}^{T}\left(\boldsymbol{Q}_{1,3}\right)^{-1}\left(\boldsymbol{y}-\tilde{\boldsymbol{E}}_{A, 2} \boldsymbol{\beta}_{2}-\left(\boldsymbol{A}-\tilde{\boldsymbol{E}}_{A, 2}\right) \hat{\boldsymbol{\beta}}_{3}\right), \\
& \tilde{\boldsymbol{e}}_{y}=\boldsymbol{Q}_{y}\left(\boldsymbol{Q}_{1,3}\right)^{-1}\left(\boldsymbol{y}-\tilde{\boldsymbol{E}}_{A, 2} \boldsymbol{\beta}_{2}-\left(\boldsymbol{A}-\tilde{\boldsymbol{E}}_{A, 2}\right) \hat{\boldsymbol{\beta}}_{3}\right)
\end{aligned}
$$

$\mathrm{Bu}$ vektörlerin elemanları Çizelge 5'de verilmektedir.

vi) Çizelge 5'de verilen hata değerlerine göre varyans bileşenleri aşağıdaki gibi hesaplanmıştır:

$$
\hat{\sigma}^{2}=\frac{\tilde{\Omega}}{n-6}=\frac{\tilde{\boldsymbol{e}}^{T} \boldsymbol{Q}_{e}^{-1} \tilde{\boldsymbol{e}}}{n-6}=\frac{\tilde{\boldsymbol{e}}_{y}^{T} \boldsymbol{Q}_{y}^{-1} \tilde{\boldsymbol{e}}_{y}+\tilde{\boldsymbol{e}}_{s}^{T} \boldsymbol{Q}_{s}^{-1} \tilde{\boldsymbol{e}}_{s}}{12-6}=0.012475937055
$$

Parametrelerin (14) eşitliği ile verilen kovaryans matrisi,

$$
\boldsymbol{C}_{\hat{\beta}}=\hat{\sigma}^{2}\left[\left(\boldsymbol{A}-\tilde{\boldsymbol{E}}_{A, 2}\right)^{T} \boldsymbol{Q}_{1,3}^{-1}\left(\boldsymbol{A}-\tilde{\boldsymbol{E}}_{A, 2}\right)\right]^{-1}
$$

biçiminde belirlenmiştir. Ötelenmiş koordinatlar ile çalış1ld1ğından, yukarıdaki kovaryans matris, " $\delta t_{1}, \delta t_{2}, k_{1}, \ldots, k_{4}$ ”. parametrelerine ilişkindir. $t_{1}$ ve $t_{2}$ öteleme parametrelerine ilişkin kovaryans matrisi elde edebilmek için, (37) eşitliklerine kovaryans yayılma kuralı uygulanarak elde edilen aşağıdaki eşitlik kullanılmalıdır;

$$
\boldsymbol{C}_{t}=\boldsymbol{H C}_{\hat{\beta}} \boldsymbol{H}^{T}, \boldsymbol{H}=\left[\begin{array}{cccccc}
1 & 0 & -u_{s} & v_{s} & 0 & 0 \\
0 & 1 & 0 & 0 & -u_{s} & -v_{s}
\end{array}\right]
$$

Söz konusu parametrelerin $\left(k_{1}, \ldots, k_{4}, \delta t_{1}, \delta t_{2}\right.$ ve de $t_{1}$ ve $t_{2}$ 'nin $)$ standart sapmaları Çizelge 6'da verilmektedir.

İteratif dengeleme işlemine göre elde edilen parametre kestirim değerlerinin ve bunların standart sapmalarının, Aydın vd. (2014)'de verilenlerle özdeş olduğu görülmektedir. Böylece, söz konusu iteratif dengeleme işleminin, sayısal olarak da WTLS çözümüne denk olduğu sonucuna varılmaktadır.

\section{Sonuç ve Öneriler}

Bu çalışmada, “TLS'nin genelleştirilmesi” (Neitzel 2010) ve "genel en küçük kareler yöntemi” (Ghilani ve Wolf 2006) birlikte düşünülerek, EIV model çerçevesinde ele alınan koordinat dönüşümü probleminin klasik dengeleme yoluyla çözümü irdelenmektedir. $\mathrm{Bu}$ amaçla oluşturulan iteratif dengeleme işleminin en küçük kareler temelli WTLS algoritmasına denk olduğu gösterilmektedir. Söz konusu iteratif dengeleme işlemi, yeni bir yöntem olarak ele alınmamalıdır.
Ancak WTLS algoritmalarına göre bir takım üstünlükleri bulunur: (1) Dönüşüm problemine ilişkin bir EIV modelin çözümünde kullanılacak WTLS algoritması için, katsayılar matrisine (A) ve hedef sistem koordinatlarına (y) ilişkin kofaktör matrise gereksinim duyulur. Bunun için, Bölüm 2'de açıklandığ1 gibi, özel bir modifikasyon işleminin göz önüne alınması gerekir. İteratif dengeleme işleminde ise, koordinatların original (verilen) kofaktör matrisleri doğrudan kullanılır. Bu nedenle, stokastik kısmın oluşturulmasında herhangi bir ek işleme gerek yoktur. (2) Herhangi bir EIV model için, hataların ve bilinmeyen parametrelerin kısmi türevlerini göz önüne alarak, iteratif dengeleme işlemine ilişkin eşitlikler kolayca çıkarılabilir. Jeodezi topluluğu, doğrusal olmayan modellerin bu şekilde çözümü için gereken doğrusallaştırma işlemine daha aşinadır. Böylesi bir dengeleme işlemi düşünüldüğünde, diğer bilinen istatistiki ve nümerik yöntemlerin EIV modele uyarlanması daha kolay bir şekilde gerçekleştirilir.

Bu çalışmada 2B Afin dönüşümü örnek olarak ele alınmıştır. Ancak, iteratif dengeleme işlemi ve iteratif kestirim sırasında ağırlık merkezine ötelenmiş koordinatların kullanılması tekniği diğer jeodezik koordinat dönüşüm problemlerine, örneğin 7 parametreli Helmert veya 9 parametreli Afin dönüşümü problemlerine kolaylıkla uyarlanabilir. Böylesi daha kapsamlı problemlerde yalnız ötelenmiş koordinatların düşünülmesi, sözü edilen nümerik sorunları ortadan kaldırmayabilir. Bunun için, hem ötelenmiş hem de normlandırılmış koordinatların kullanılması gerekebilir: Böylesi bir durumda, açıklanan iteratif dengeleme eşitlikleri değişmez, ancak, öteleme parametrelerinin aranan değerlerinin her bir iterasyon sonrasında uygun eşitlikler yardımıyla nasıl elde edileceğini düşünmek gerekir.

Çizelge 6. Parametre kestirim değerlerinin standart sapmalar

\begin{tabular}{|c|c|}
\hline Parametre & Standart Sapma \\
\hline $\mathrm{k}_{1}$ & 0,000011320243 \\
\hline $\mathrm{k}_{2}$ & 0,000011032937 \\
\hline $\mathrm{k}_{3}$ & 0,000015787378 \\
\hline $\mathrm{k}_{4}$ & 0,000013057698 \\
\hline$\delta \mathrm{t}_{1}(\mathrm{~m})$ & 0,031875120007 \\
\hline$\delta \mathrm{t}_{2}(\mathrm{~m})$ & 0,041893765662 \\
$\mathrm{t}_{1}(\mathrm{~m})$ & 0,121461424911 \\
$\mathrm{t}_{2}(\mathrm{~m})$ & 0,167012387036 \\
\hline
\end{tabular}




\section{Ek A}

$n \times n(n=2 p)$ boyutlu

$$
\begin{aligned}
& \boldsymbol{D}_{j}=\boldsymbol{I}_{p} \otimes \boldsymbol{K}_{j} \\
& \left(\begin{array}{l}
j=3,4,5,6 \text { and } \\
\left.\boldsymbol{K}_{3}=\left[\begin{array}{ll}
1 & 0 \\
0 & 0
\end{array}\right], \boldsymbol{K}_{4}=\left[\begin{array}{cc}
0 & -1 \\
0 & 0
\end{array}\right], \boldsymbol{K}_{5}=\left[\begin{array}{ll}
0 & 0 \\
1 & 0
\end{array}\right], \boldsymbol{K}_{6}=\left[\begin{array}{ll}
0 & 0 \\
0 & 1
\end{array}\right]\right)
\end{array}\right.
\end{aligned}
$$

permütasyon matrisleriyle $\boldsymbol{e}_{s}$ rasgele hata vektörü, $\mathbf{E}_{A}$ hata matrisinin vektörel haline aşağıdaki biçimde dönüştürülür;

$\boldsymbol{e}_{A}=\operatorname{vec} \boldsymbol{E}_{A}=\left[\begin{array}{c}\boldsymbol{0} \\ \boldsymbol{0} \\ \boldsymbol{D}_{3} \\ \boldsymbol{D}_{4} \\ \boldsymbol{D}_{5} \\ \boldsymbol{D}_{6}\end{array}\right]=\boldsymbol{J} \boldsymbol{e}_{s}$

Böylece, $6 n \times n$ boyutlu $\mathbf{J}$ matrisi tanımlanır.

\section{Ek B: Korelasyonlu gözlemler için en küçük kareler te- melli WTLS algoritması}

$$
\begin{aligned}
& \hat{\boldsymbol{\beta}}_{0} \text { ve } \tilde{\boldsymbol{E}}_{A, 0} \text { başlangıç değerlerini tespit et (bk. (13) eşitliği). } \\
& j=1,2 \ldots \text { için tekrarla: } \\
& \boldsymbol{L}_{j}=\hat{\boldsymbol{\beta}}_{j-1} \otimes \boldsymbol{I}_{n}, \boldsymbol{Q}_{1, j}=\boldsymbol{Q}_{y}-\boldsymbol{Q}_{y A} \boldsymbol{L}_{j}-\boldsymbol{L}_{j}^{T} \boldsymbol{Q}_{A y}+\boldsymbol{L}_{j}^{T} \boldsymbol{Q}_{A} \boldsymbol{L}_{j}, \boldsymbol{P}_{1, j}=\boldsymbol{Q}_{1, j}^{-1} \\
& \tilde{\boldsymbol{A}}_{j}=\boldsymbol{A}-\tilde{\boldsymbol{E}}_{A, j-1}, \tilde{\boldsymbol{y}}_{j}=\boldsymbol{y}-\tilde{\boldsymbol{E}}_{A, j-1} \hat{\boldsymbol{\beta}}_{j-1}, \hat{\boldsymbol{\beta}}_{j}=\left(\tilde{\boldsymbol{A}}_{j}^{T} P_{1, j} \tilde{\boldsymbol{A}}_{j}\right)^{-1} \tilde{\boldsymbol{A}}_{j}^{T} P_{1, j} \tilde{\boldsymbol{y}}_{j} \\
& \tilde{\boldsymbol{\beta}}_{A, j}=\left(\boldsymbol{Q}_{A y}-\boldsymbol{Q}_{A}\left(\hat{\boldsymbol{\beta}}_{j} \otimes \boldsymbol{I}_{n}\right)\right) \boldsymbol{P}_{1, j}\left(\tilde{\boldsymbol{y}}_{j}-\tilde{\boldsymbol{A}}_{j} \hat{\boldsymbol{\beta}}_{j}\right) \text { ve } \widetilde{\mathbf{E}}_{\mathrm{A} \mathrm{j}}=\operatorname{vec}^{-1}\left(\widetilde{\mathbf{e}}_{\mathrm{A} \mathrm{j}}\right) \\
& " \max \left(\left|\hat{\boldsymbol{\beta}}_{j}-\hat{\boldsymbol{\beta}}_{j-1}\right|\right) " \text { karş1laştırma değerinden küçük oluncaya dek ite- } \\
& \operatorname{rasyona~devam~et.~}
\end{aligned}
$$

\section{Kaynaklar}

Amiri-Simkooei, A. and Jazaeri, S., (2012), Weighted total leastsquares formulated by standard least squares theory, Journal of Geodetic Science 2(2): pp.113-124.
Aydın, C., Uygur M., Uygur S. Ö., (2014), Ağırlıklı toplam en küçük kareler çözümü: Üç farklı algoritma ve 2-boyutlu Afin dönüşümüne uygulanması, Harita Dergisi, Sayı 152, 1-11.

Carroll, R.J. and Ruppert, D., (1996), The use and misuse of orthogonal regression in linear errors-in-variables models, The American Statistician, 50(1): pp. 1-6.

Fang, X., (2011), Weighted total least squares solutions for applications in geodesy, $\mathrm{PhD}$ Dissertation, Geodaesie und Geoinformatik Der Leibniz Universitaet Hannover, Hannover.

Fang, X., (2013), Weighted total least squares: necessary and sufficient conditions, fixed and random parameters, Journal of Geodesy, 87: pp. 733-749.

Ghilani, C.D. and Wolf, P.R., (2006), Adjustment computations: Spatial Data Analysis, Fourth Edition, John Wiley\&Sons Inc., Hoboken, New Jersey.

Golub, G.H. and van Loan, C.F., (1980), An analysis of the total least squares problem, SIAM J. Numer. Anal., 17(6): pp. 883893.

Koch, K.R., (1999), Parameter estimation and hypothesis testing in linear models, Second Edition, Springer-Verlag, Berlin/ Heidelberg.

Neitzel, F., (2010), Generalization of total least-squares on example of unweighted and weighted $2 D$ similarity transformation, Journal of Geodesy, 84(12): pp. 751-762.

Pope, A. J., (1972), Some pitfalls to be avoided in the iterative adjustment of nonlinear problems, In: Proceedings of the 38th Annual Meeting of the American Society of Photogrammetry, Washington DC: pp. 449-477.

Schaffrin, B. and Wieser, A., (2008), On weighted total leastsquares adjustment for linear regression, Journal of Geodesy, 82: pp. 415-421.

Schaffrin, B. and Snow, K., (2010), Total least-squares regularization of Tykhonov type and an ancient racetrack in Corinth, Linear Algebra and its Applications, 432(8): pp. 20612076.

Snow, K., (2012), Topics in total least-squares adjustment within the errors-in-variables model: Singular cofactor matrices and prior information, Report No. 502, Geodetic Science Ohio State University, Columbus, Ohio.

Tong, X., Jin, Y., Li, L., (2011), An improved weighted total least squares method with applications in linear fitting and coordinate transformation, Journal of Surveying Engineering, 137(4): pp. 120-128.

Van Huffel, S., (2004), Total least-squares and errors-in-variables modeling: Bridging the gap between statistics, computational mathematics and engineering, COMPSTAT 2004 Symposium, Physica-Verlag/Springer: pp. 1-16. 